\title{
新型给体-受体型聚合物的合成及其在本体异质结聚合物 太阳能电池中的应用
}

\author{
邹文武 $a$ 刘 颖 ${ }^{b}$ 贾庆明*, $a$ 葛子义 $*, b$ \\ ( ${ }^{a}$ 昆明理工大学化学工程学院 昆明 650500) \\ ( ${ }^{b}$ 中国科学院宁波材料技术与工程研究所 宁波 315201)
}

\begin{abstract}
摘要 设计、合成了侧链含有强吸电结构的丙二酸二丁酯受体单元与苯并 $\left[1,2-b: 4,5-b^{\prime}\right]$ 二噻吩给体单元交替共聚物 PBDTDT, 研究了其热学、光学、电化学性质以及与受体 $\mathrm{PC}_{71} \mathrm{BM}$ ([6,6]-苯基 $\mathrm{C}_{71}$ 丁酸甲酯)共混作为活性层制备成本体 异质结聚合物有机太阳能电池的光伏性质, 考察了 PBDTDT 与 $\mathrm{PC}_{71} \mathrm{BM}$ 不同比例时的光伏性能, 当聚合物 PBDTDT 和 $\mathrm{PC}_{71} \mathrm{BM}$ 质量比为 $1: 3$ 制备的器件, 其开路电压达到了 $0.82 \mathrm{~V}$, 能量转换效率 $(\mathrm{PCE})$ 为 $0.90 \%$, 短路电流为 $3.25 \mathrm{~mA} / \mathrm{cm}^{2}$, 填充因子 $\mathrm{FF}$ 为 0.338 , 同时将其与同等工艺制备的 poly(3-hexylthiophene) (P3HT)太阳能电池的光伏性能进行比较, 相 同工艺下制备的 P3HT 电池的开路电压仅为 $0.55 \mathrm{~V}$, 由 PBDTDT 制备的电池开路电压比 P $3 \mathrm{HT}$ 电池的开路电压高出 0.29 $\mathrm{V}$, 同时分析了 PBDTDT 能量转换效率较 P3HT 低的原因.
\end{abstract}

关键词＼cjkstart丙二酸二丁酯; 交替共聚物; 有机太阳能电池;

\section{Synthesis of New Donor-Acceptor Copolymer and Its Application in Organic Bulk Hetero-junction Solar Cells}

\author{
Zou, Wenwu $^{a} \quad$ Liu, Ying $^{b} \quad$ Jia, Qingming*,a $\quad$ Ge, Ziyi* ${ }^{*, b}$ \\ $\left({ }^{a}\right.$ Faculty of Chemical Engineering, Kunming University of Science and Technology, Kunming Yunnan Province, \\ Kunming 650500) \\ ( ${ }^{b}$ Ningbo Institute of Materials Technology \& Engineering, Chinese Academy of Sciences, Ningbo 315201)
}

\begin{abstract}
Alternative copolymer (PBDTDT) was synthesized from acceptor unit with strong electro-withdrawing structure of dibutyl malonate in branch chain and donor unit based on benzo[1,2-b:4,5- $\left.b^{\prime}\right]$ dithiophene. And its thermal, optical, electrochemical properties and photovoltaic properties blending with $[6,6]$-phenyl- $\mathrm{C}_{71}$ butyric acid methyl ester as the active layer in the bulk hetero-junction devices of organic solar cells were investigated. The influence of different ratio of polymer PBDTDT and $\mathrm{PC}_{71} \mathrm{BM}$ on the photovoltaic properties was also studied. And the open voltage reached $0.82 \mathrm{~V}$, when the weight ratio of PBDTDT and $\mathrm{PC}_{71} \mathrm{BM}$ is $1: 3$, and its power conversion efficiency (PCE) was $0.90 \%$, with $V_{\mathrm{oc}}$ of $0.82 \mathrm{~V}, J_{\mathrm{sc}}$ of $3.25 \mathrm{~mA} / \mathrm{cm}^{2}$ and FF of 33.8\%. Comparing with the poly(3-hexylthiophene) (P3HT) in the same method, the solar cells with P3HT blending with $\mathrm{PC}_{71} \mathrm{BM}$ as the active layer can only get $0.55 \mathrm{~V}$, which can be lower about $0.29 \mathrm{~V}$ than that of the PBDTDT. And the possible factors resulting in low PCE and the higher open voltage than that of P3HT were analyzed.
\end{abstract}

Keywords dibutyl malonate; alternative copolymer; organic solar cells

随着全球对能源需求增加以及对环境要求的提高, 发展低廉、清洁的能源已迫在眉睫, 充分利用太阳能资 源来解决能源危机成为一条切实有效的途径, 与无机太 阳能电池相比，聚合物太阳能电池因其具有制备简单、 成本低、质量轻、可制备成柔性器件等优点，而受到广
泛关注 ${ }^{[1 \sim 4]}$. 目前聚 3-己基噻吩(P3HT), 因其具有良好 的溶解性、规整性和成膜性, 已被广泛研究. 但由于聚 3 -已基噻吩的 $\mathrm{HOMO}$ 能级较高, 达到了 $-5.17 \mathrm{eV}^{[5]}$, 常 见受体 $\mathrm{PC}_{61} \mathrm{BM}$ 的最低空轨道(LUMO) 能级较低, 在 $\mathrm{P} 3 \mathrm{HT}$ 与 $\mathrm{PC}_{61} \mathrm{BM}$ 制备的太阳能电池的器件中, 最高开

\footnotetext{
*E-mail: jiaqm411@163.com

Received December 12, 2012; revised February 23, 2013; published online March 1, 2013.

Project supported by the National Natural Science Foundation of China (No. 21074144).

国际自然科学基金(No. 21074144)资助项目.
} 
路电压仅能达到 $0.58 \mathrm{~V}^{[6]}$, 限制了聚 3-已基噻吩太阳能 电池的能量转换效率的提高. 有机聚合物因对太阳光光 谱吸收的波谱较窄, 不能与太阳光谱相匹配, 导致所制 备太阳能电池的能量转化效率(PCE) 普遍较低, 通过将 吸电子结构引入到受体单元，与给电子给体单元交替共 聚组成给体一受体(Donor-Acceptor, D-A) 型聚合物 ${ }^{[7 \sim 9]}$, 达到对太阳光发射光谱吸收红移, 使聚合物的吸收峰尽 量与太阳光发射光谱匹配, 亦可通过扩宽对太阳能光谱 的吸收 ${ }^{[10,11]}$, 对活性层进行热退火 ${ }^{[12]}$ 以及加入添加 剂 ${ }^{[13]}$ 来提高电池的能量转化效率. 目前聚合物太阳能 电池的 PCE 已超过 $9 \%{ }^{[4]}$. 本文设计了含有苯并 [1,2-b:4,5- $\left.b^{\prime}\right]$ 二噻吩作为给体单元与丙二酸二丁酯作为 侧链的受体单元进行交替共聚制备的新型 D-A 聚合物, 并对该聚合物与 $\mathrm{PC}_{71} \mathrm{BM}$ 不同质量比制备的太阳能电池 进行了优化, 得到最佳条件为聚合物和 $\mathrm{PC}_{71} \mathrm{BM}$ 质量比 为 $1: 3$, 添加剂为体积比 $2 \%$ 的 1,8 -二碘辛烷. 在这种条 件下所得器件的开路电压达到了 $0.82 \mathrm{~V}$, 能量转换效率 (PCE) 为 $0.90 \%$, 短路电流为: $3.25 \mathrm{~mA} / \mathrm{cm}^{2}$, 填充因子 $\mathrm{FF}$ 为 0.338 .

\section{1 结果与讨论}

\section{1 聚合物的结构表征}

\subsection{1 聚合物的热学性质}

聚合物的数均分子量 $\left(M_{\mathrm{n}}\right)$, 质均分子量 $\left(M_{\mathrm{w}}\right)$, 聚合 物分散指数(PDI) 以及分解温度 $\left(T_{\mathrm{d}}\right)$ 和玻璃化转化温度 $\left(T_{\mathrm{g}}\right)$ 列于下表 1 , 其中聚合物的分解温度是在氮气氛围 下, 以 $10{ }^{\circ} \mathrm{C} / \mathrm{min}$ 的速度下进行测量的. 而玻璃化转变 温度 $\left(T_{\mathrm{g}}\right)$ 则是通过差示扫描量热仪进行测试获得的. 从 下表 1 中可以看出, 该聚合物的分解温度达到了 368.5 ${ }^{\circ} \mathrm{C}$, 表现出良好的热稳定性.

表 1 聚合物的分子量与热学性质

Table 1 Molecular weight and thermal properties of polymer

\begin{tabular}{ccccc}
\hline$M_{\mathrm{n}} /\left(\mathrm{kg} \cdot \mathrm{mol}^{-1}\right)$ & $M_{\mathrm{w}} /\left(\mathrm{kg} \cdot \mathrm{mol}^{-1}\right)$ & PDI & $T_{\mathrm{d}}{ }^{\circ} \mathrm{C}$ & $T_{\mathrm{g}} /{ }^{\circ} \mathrm{C}$ \\
\hline 6.808 & 16.786 & 2.46 & 368.5 & 86.8 \\
\hline
\end{tabular}

\subsection{2 聚合物的光学性质}

图 1 为聚合物在氯仿溶液和薄膜中的紫外-可见吸 收光谱图, 在薄膜中, 聚合物在 $423 \mathrm{~nm}$ 有较强的吸收, 此为聚合物的 $\pi-\pi$ 共轭重叠引起的吸收, 而 $525 \mathrm{~nm}$ 处有 强吸收峰, 应为主链上给体单元与受体单元的分子内的 电荷相互作用引起的吸收. 薄膜中的紫外可见吸收光谱 较溶液中的紫外可见吸收谱图红移了约 $40 \mathrm{~nm}$, 但仍与 太阳发射光谱不太匹配.

\subsection{3 聚合物的电化学性质}

聚合物的最高占有轨道(HOMO)和最低空轨道

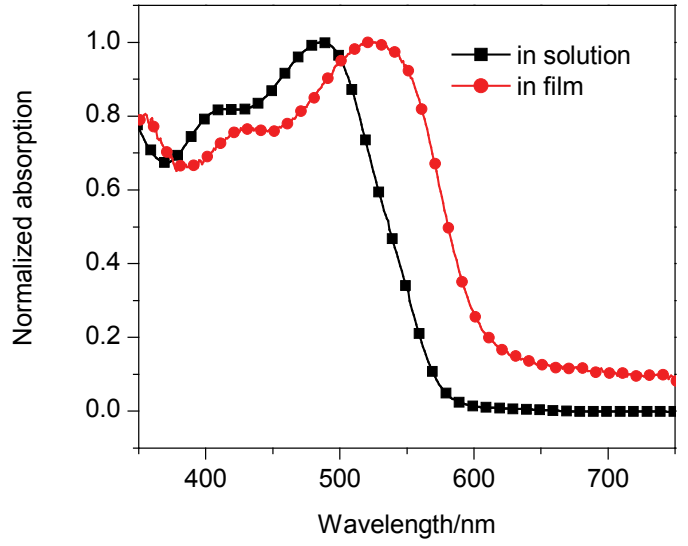

图 1 聚合物在氯仿溶液及在薄膜中的归一化紫外-可见吸收 谱图

Figure 1 Normalized UV-Vis absorption spectra of polymer in chloroform solution and in thin films.

(LUMO) 是在 ITO 基片上涂上聚合物作为工作电极, $\mathrm{Ag} / \mathrm{AgCl}$ 为参比电极以及辅助电极浸在 $0.1 \mathrm{~mol} / \mathrm{L}$ $\mathrm{Bu}_{4} \mathrm{NPF}_{6}$ 的乙腈溶液中进行测量的, 其中的参比电极是 在真空下用 $\mathrm{Fc} / \mathrm{Fc}^{+}$还原对进行校准. 聚合物的 $\mathrm{HOMO}$ 能级与 LUMO 能级采用经验公式 $\mathrm{HOMO}=-\left(E_{\mathrm{on}}^{\mathrm{ox}}-\right.$ $\left.E_{\text {on }}^{\text {ferrocene }}+4.80\right)(\mathrm{eV}), \quad \mathrm{LUMO}=-\left(E_{\mathrm{on}}^{\text {red }}-E_{\mathrm{on}}^{\text {ferrocene }}+\right.$ $4.80)(\mathrm{eV})^{[14]}$ 计算得出的, 其中 $4.80 \mathrm{eV}$ 是二茂铁在真空 下的能级. $E_{\mathrm{on}}^{\mathrm{ox}}$ 和 $E_{\mathrm{on}}^{\mathrm{red}}$ 为以参比电极作为标准的氧化、 还原电位. 聚合物的 HOMO 与 LUMO 能级列于表 2.

表 2 聚合物的电化学性质

Table 2 Electrochemical energy of polymer

\begin{tabular}{ccccccc}
\hline \multirow{2}{*}{ Polymer } & \multicolumn{2}{c}{$\lambda / \mathrm{nm}$} & \multirow{2}{*}{$E_{\text {Hомо }}$} & $E_{\mathrm{LUMO}}$ & \multirow{2}{*}{$E_{\mathrm{g}}{ }^{a} / \mathrm{eV}$} & \multirow{2}{*}{$E_{\mathrm{g}}{ }^{b} / \mathrm{eV}$} \\
\cline { 2 - 6 } & Solution & Film & & & \\
\hline PBDTDT & 550 & 615 & -5.55 & -3.69 & 2.01 & 1.86 \\
\hline
\end{tabular}

${ }^{a}$ Estimated from the onset wavelength absorptions of the solid films. ${ }^{b}$ Estimated from the onset of corresponding potential

\section{2 聚合物太阳能电池的制备及其性能的测定}

聚合物太阳能电池的结构为: ITO/PEDOT:PSS/ polymer:PC $\mathrm{PC}_{71} \mathrm{BM} / \mathrm{LiF} / \mathrm{Al}$. 其中 $\mathrm{ITO}$ 基片依次用去离子 水、丙酮、异丙醇超声波清洗 $20 \mathrm{~min}$ 后，在氮气的气流 中干燥, 备用. PEDOT:PSS 过滤后以 $4000 \mathrm{r} / \mathrm{min}$ 转速旋 涂于 ITO 基片上的. 聚合物和 $\mathrm{PC}_{71} \mathrm{BM}$ 以不同的质量比 分别溶于氯仿与邻二氯苯 $(V: V=1: 3)$ 组成的混合溶 剂, 在加热条件下搅拌, 待溶解之后, 在手套箱中通过 旋涂仪将以 $1000 \mathrm{r} / \mathrm{min}$ 的速度旋涂于 ITO 上, 然后在真 空蒸镀室于 $1 \times 10^{-4} \mathrm{~Pa}$ 条件下, 将 $\mathrm{LiF}$ 和 $\mathrm{Al}$ 电极依次蒸 镀到活性层表面，两者的厚度分别为 1 和 $100 \mathrm{~nm}$, 制成 的电池有效面积为 $0.1257 \mathrm{~cm}^{2}$, 聚合物太阳能电池能量 转换效率则是在模拟太阳光强为 $1.5 \mathrm{AM}$ 的条件下, 通 过 Keithly 2400 电流电压测试仪进行测定. 
聚合物与 $\mathrm{PC}_{71} \mathrm{BM}$ 不同质量比例制备的器件所得的 $\mathrm{PCE}$ 如图 2 所示, 在聚合物与 $\mathrm{PC}_{71} \mathrm{BM}$ 质量比为 $1: 3$, 添加剂为 $2 \%$ 体积比 1,8 -二碘辛烷(DIO)条件下, 所得器 件 PCE 达到 $0.90 \%$, 开路电压 $V_{\mathrm{oc}}$ 为 $0.82 \mathrm{~V}$, 短路电流 $J_{\mathrm{sc}}$ 达到 $3.25 \mathrm{~mA} / \mathrm{cm}^{2}$, 填充因子 $\mathrm{FF}$ 为 0.338 , 可能是随 着 $\mathrm{PC}_{71} \mathrm{BM}$ 的含量增加, 增加了激子分离与传导, 从而 提高到了器件的短路电流. 图 3 是聚合物光伏电池活性 层形貌的原子力显微镜(AFM)图, 从图中可以看出, $\mathrm{a}, \mathrm{b}$ 两图出现了聚集, 较为粗粘, 相比 $\mathrm{a}, \mathrm{b}$ 图, $\mathrm{c}$ 图的表面形 貌具有更加平滑的表面, 没有出现明显的相分离, 有利 于增加电荷的传导, 提高电流, 这与相应器件的电流最 高也是一致的. 但可能因 $\mathrm{PC}_{71} \mathrm{BM}$ 过于均匀分散在聚合 物中, 导致聚合物和 $\mathrm{PC}_{71} \mathrm{BM}$ 之间的相分离较小, 使所 产生的激子不能有效分离, 同时由于传输路径不连续, 使得已经生成的载流子复合的概率增大, 减小了载流子 的寿命, 造成了能量的损失 ${ }^{[15,16]}$, 导致了器件 PCE 较低.

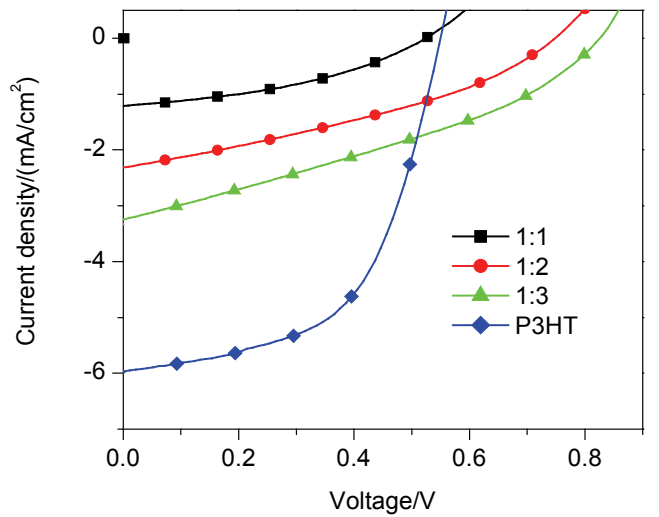

图 2 聚合物与 $\mathrm{PC}_{71} \mathrm{BM}$ 质量比为: $1: 1,1: 2,1: 3$ 以及 $\mathrm{P} 3 \mathrm{HT}$ 的光伏电池 $J-V$ 曲线

Figure $2 J-V$ curves of photovoltaic cells based on polymer (weight ratio ranging from $1: 1$ to $1: 3$ with $\mathrm{P}_{71} \mathrm{CBM}$ ) and the $\mathrm{P} 3 \mathrm{HT}$ (weight ratio was $1: 1$ corresponding to $\mathrm{PC}_{71} \mathrm{BM}$ ), respectively
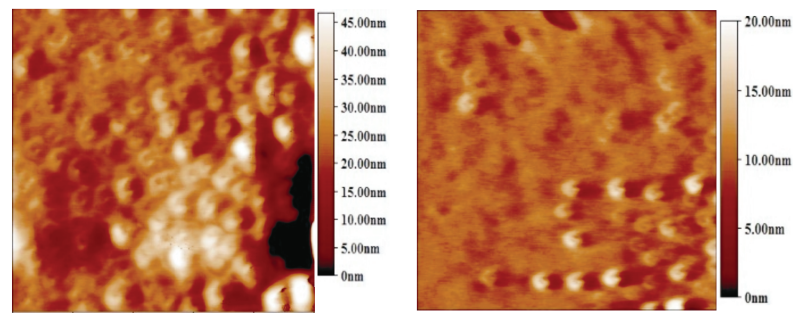

图 $3 \mathrm{a}, \mathrm{b}, \mathrm{c}$ 分别是聚合物和 $\mathrm{PC}_{71} \mathrm{BM}$ 比例为 $1: 1,1: 2,1: 3$ 时的 AFM 图

Figure 3 The AFM image of weight ratio ranging from $1: 1$ to $1: 3$ for polymer and $\mathrm{PC}_{71} \mathrm{BM}$, respectively

相同条件下制备的 P3HT 聚合物太阳能电池效率达 到了 $1.83 \%$, 其中 $\mathrm{P} 3 \mathrm{HT}$ 与 $\mathrm{PC}_{71} \mathrm{BM}$ 质量比为 $1: 1$ 添加 剂体积比 $1 \%$ 的 1,8-辛二硫醇. 聚合物 PBDTDT 能量转
换效率相对比较低，可能是因为聚合物自身对太阳光谱 的吸收较弱. 在聚合物旋涂退火完毕后, 所得器件仍较 透明, 而由 P3HT 制备的器件则表现出颜色加深的趋势, 退火使 P3HT 主链间规整度增加，增加了 $\pi-\pi$ 堆积，有利 于对光谱的吸收，提高聚合物的电流. 与 P3HT 相比, 聚合物 PBDTDT 有着相对较高的开路电压，达到了 0.82 $\mathrm{V}$, 相对应的 P3HT 则仅仅只有 $0.55 \mathrm{~V}$. 可能由于 P3HT 的 HOMO 能级为 $-5.17 \mathrm{eV}^{[5]}$, 而 PBDTDT 的 HOMO 能 级则是在 $-5.55 \mathrm{eV}$, 在相同受体 $\mathrm{PC}_{71} \mathrm{BM}$ 的条件下，则 聚合物的 $\mathrm{HOMO}$ 能级与 $\mathrm{PC}_{71} \mathrm{BM}$ 的 $\mathrm{LUMO}$ 能级之差大 于 $\mathrm{P} 3 \mathrm{HT}$ 的 $\mathrm{HOMO}$ 能级与 $\mathrm{PC}_{71} \mathrm{BM}$ 的能级差, 根据聚合 物太阳能电池的开路电压与聚合物 HOMO 能级与 $\mathrm{PC}_{71} \mathrm{BM}$ 的 LUMO 能级差是呈正比的 ${ }^{[17,18]}$, 故所得聚合 物太阳能电池的开路电压相对 P3HT 制备的太阳能电 池，有着更高的开路电压. 相信通过将丙二酸酯结构引 入到聚合物太阳能电池中, 有助于提高聚合物的开路电 压.

\section{2 结论}

本文通过简便方法合成了强吸电子受体单元，制备 了主链为给一受体结构的共轭聚合物, 将聚合物与受体 材料 $\mathrm{PC}_{71} \mathrm{BM}$ 共混后制备的太阳能电池, 在聚合物和 $\mathrm{PC}_{71} \mathrm{BM}$ 混合比为 $1: 3$, 以 $2 \%$ 体积比的 DIO 作为添加 剂时, 太阳能电池开路电压达到了 $0.82 \mathrm{~V}$, 能量转换效 率(PCE) 为 $0.90 \%$, 短路电流为: $3.25 \mathrm{~mA} / \mathrm{cm}^{2}$, 填充因子 $\mathrm{FF}$ 为 0.338 , 同时与最具代表性的光伏材料 P3HT 制备 的太阳能电池做了比较, 聚合物的开路电压比 P3HT 高 了 $0.29 \mathrm{~V}$.

\section{3 实验部分}

\section{1 仪器与试剂}

试剂均购于 Aldrich 或者 J\&K 百灵威公司, 无水四 氢呋喃(THF)是在二苯甲酩作为指示剂、钠丝存在下, 回 流除水. $N, N$ 二 二甲基甲酰胺(DMF)则是在无水硫酸镁干 燥后，真空蒸馏得到的. 德国 Bruker 公司 DMX400 型核 磁共振仪 $\left({ }^{1} \mathrm{H}\right.$ NMR, ${ }^{13} \mathrm{C}$ NMR), UV-2401PC Shimadzu 紫 外一可见光谱仪, Waters 2695 凝胶渗透色谱仪(GPC)以 THF 作为流动相, 线性单分散聚苯乙烯标样. Pyris-1 热 重分析仪(氮气流速 $20 \mathrm{~mL} / \mathrm{min}$, 升温速率为 $10{ }^{\circ} \mathrm{C} / \mathrm{min}$ ), 原子力显微镜(本原纳米仪器有限公司), Dektak 150 stylus surface profiler 膜厚仪.

\section{2 实验方法}

\section{2 .1 单体的合成}

合成路线如 Scheme 1 所示.

2,5-二溴噻吩-3-甲醛(1)根据文献[19]改进合成. 在 
$250 \mathrm{~mL}$ 三口瓶中，加入 3-噻吩甲醛基(4.48 g $10 \mathrm{mmol}$ )、 加入二氯甲烷 $(100 \mathrm{~mL})$ 、碳酸氢钠 $(4.5 \mathrm{~g}, 53 \mathrm{mmol})$, 在剧 烈搅拌下, 溴素 $(7.5 \mathrm{~g}, 46 \mathrm{mmol})$ 通过恒压漏斗逐滴加入 到三口瓶中, 反应 $24 \mathrm{~h}$ 后, 加入硫代硫酸钠除去过量的 溴素, 直到反应溶液颜色变浅. 将反应混合物倒入去离 子水中, 用二氯甲烷萃取 3 次, 无水硫酸镁干燥. 硅胶 柱层析纯化(正己烷/乙酸乙酯, $V: V=10: 1$ ). 得到棕 色固体(4.23 g, 产率 39.3\%). ${ }^{1} \mathrm{H}$ NMR (400 MHz, $\mathrm{CDCl}_{3}$ ) $\delta: 9.79(\mathrm{~s}, 1 \mathrm{H}), 7.33(\mathrm{~s}, 1 \mathrm{H})$.

2-(2,5-二溴噻吩-3-乙烯基)丙二酸二丁酯(2)根据文 献[20]改进合成的. 在 $50 \mathrm{~mL}$ 三口瓶中加入 2,5-二溴 3醛基噻吩 $(2.69 \mathrm{~g}, 10 \mathrm{mmol})$ 、丙二酸二丁酯 $(2.16 \mathrm{~mL}, 10$ $\mathrm{mmol}) 、$ 哌啶 $(0.1 \mathrm{~mL})$, 在氩气氛围下, 反应回流 $5 \mathrm{~h}$, 减 压旋出乙腈, 用二氯甲烷萃取 3 次, 无水硫酸镁干燥, 通过硅胶柱层析纯化(正已烷/乙酸乙酯, $V: V=10: 1$ ), 产物为淡黄色液体 $(0.85 \mathrm{~g}$, 产率 $45 \%)$. ${ }^{1} \mathrm{H}$ NMR (400 $\left.\mathrm{MHz}, \mathrm{CDCl}_{3}\right) \delta: 7.58(\mathrm{~s}, 1 \mathrm{H}), 7.01(\mathrm{~s}, 1 \mathrm{H}), 4.28 \sim 4.30(\mathrm{~m}$,
$2 \mathrm{H}), 4.22 \sim 4.25(\mathrm{~m}, 2 \mathrm{H}), 1.62 \sim 1.73(\mathrm{~m}, 4 \mathrm{H}), 1.34 \sim 1.45$ $(\mathrm{m}, 4 \mathrm{H}), 0.92 \sim 0.96(\mathrm{~m}, 6 \mathrm{H}) ;{ }^{13} \mathrm{C}$ NMR $(400 \mathrm{MHz}$, $\left.\mathrm{CDCl}_{3}\right) \delta: 166.09,163.81,134.51,131.90,128.54,126.54$, $119.20,112.69,77.46,77.17,76.82,65.73,30.51,29.67$, $19.14,13.72$.

化合物 4 根据文献[21]合成, 通过硅胶柱层析纯化 $[V($ 石油醚 $): V$ (三乙胺 $)=100: 5]$ 得到浅黄色粘稠液体 $1.1 \mathrm{~g}$, 产率 47\%. ${ }^{1} \mathrm{H}$ NMR $\left(400 \mathrm{MHz}, \mathrm{CDCl}_{3}\right) \delta: 7.7(\mathrm{t}$, $J=24 \mathrm{~Hz}, 2 \mathrm{H}), 7.34(\mathrm{~d}, J=4 \mathrm{~Hz}, 2 \mathrm{H}), 6.89 \sim 6.90(\mathrm{~m}$, $2 \mathrm{H}), 2.87(\mathrm{~d}, J=7 \mathrm{~Hz}, 4 \mathrm{H}), 1.54 \sim 1.61(\mathrm{~m}, 16 \mathrm{H}), 1.34 \sim$ $1.45(\mathrm{~m}, 26 \mathrm{H}), 1.29 \sim 1.40(\mathrm{~m}, 10 \mathrm{H}), 0.87 \sim 0.97(\mathrm{~m}, 32 \mathrm{H})$. 3.2.2 聚合物的合成

取 $25 \mathrm{~mL}$ 干燥三口瓶，加入化合物 $2(140 \mathrm{mg}, 0.3$ $\mathrm{mmol}$ )和化合物 $4(347 \mathrm{mg}, 0.3 \mathrm{mmol})$, 加入无水甲苯 12 $\mathrm{mL}$ 、无水 $N, N$-二甲基甲酰胺 $3 \mathrm{~mL}$, 在氮气保护下, 加 入 $\mathrm{Pd}\left(\mathrm{PPh}_{3}\right)_{4}(35 \mathrm{mg}, 30 \mathrm{mmol})$, 反应在 $120{ }^{\circ} \mathrm{C}$ 下进行 48 $\mathrm{h}$, 反应结束后，将反应液逐滴加到甲醇中，过滤，依次
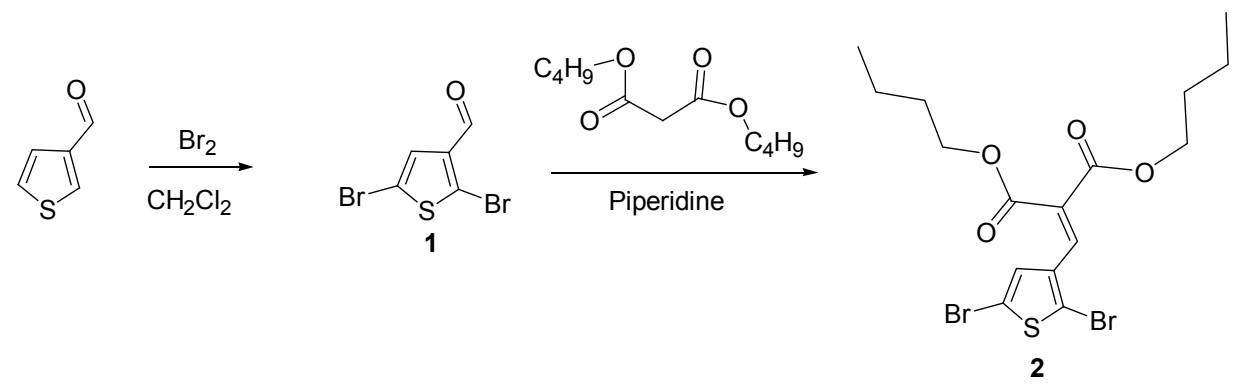

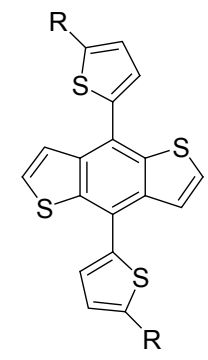

3

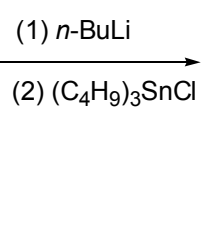

(2) $\left(\mathrm{C}_{4} \mathrm{H}_{9}\right)_{3} \mathrm{SnCl}$

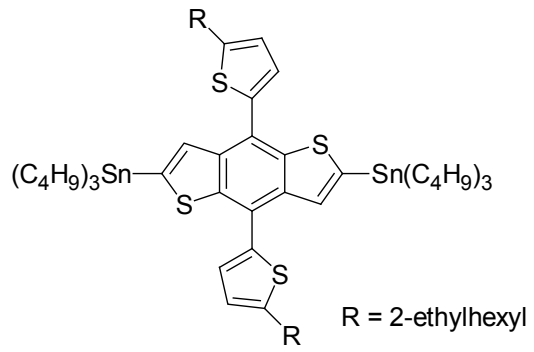

4

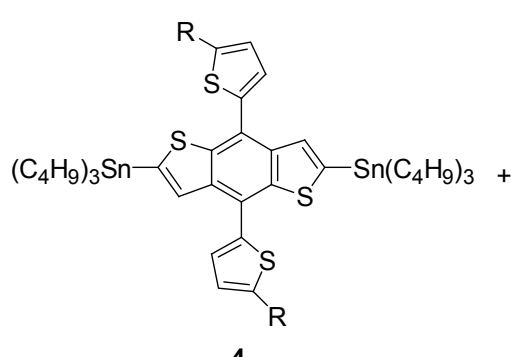

4

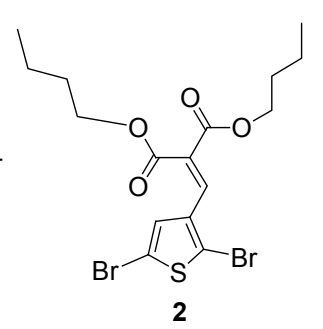

2
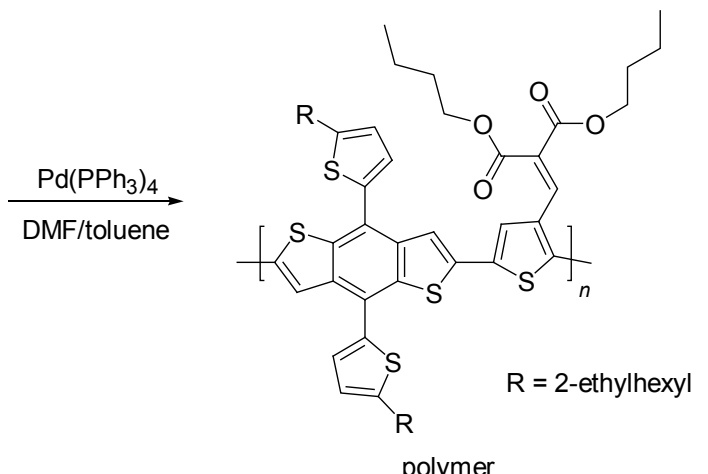

Scheme 1 
用甲醇、正己烷、氯仿抽提, 将氯仿旋至少量之后, 逐 滴滴入到甲醇中, 过滤, 将产物干燥, 得到深红色固体 $135.5 \mathrm{mg}$, 产率 51\%. ${ }^{1} \mathrm{H}$ NMR (400 MHz, $\left.\mathrm{CDCl}_{3}\right) \delta: 7.98$ (br, 1H), 7.69 (br, 3H), 7.38 (br, 1H), 7.31 (br, 2H), 6.92 (br, 2H), 4.28 (br, 4H), 2.88 (br, 4H), 1.69 (br, 6H), 1.36 (br, 20H), 0.928 (br, 18H).

\section{References}

[1] Chu, T.; Lu, J.; Beaupré, S.; Zhang, Y.; Pouliot, J.; Wakim, S.; Zhou, J.; Leclerc, M.; Li, Z.; Ding, J.; Tao, Y. J. Am. Chem. Soc. 2011, 133, 4250 .

[2] Li, W.; Roelofs, W. S. C.; Wienk, M. M.; Janssen, R. A. J. J. Am. Chem. Soc. 2012, 134, 13787.

[3] Hou, J.; Chen, H.; Zhang, S.; Chen, R. I.; Yang, Y.; Wu, Y.; Li, G. J. Am. Chem. Soc. 2009, 131, 15586.

[4] Peng, Q.; Huang, Q.; Hou, X.; Chang, P.; Xu, J.; Deng, S. Chem. Commun. 2012, 48, 11452.

[5] Burkhart, B.; Khlyabich, P. P.; Thompson, B. C. Macromolecules 2012, 45, 3740 .

[6] He, Y.; Chen, H.; Hou, J.; Li, Y. J. Am. Chem. Soc. 2010, 132, 1377.

[7] Zhang, M.; Guo, X.; Li, Y. Macromolecules 2011, 44, 8798.

[8] Huo, L.; Ye, L.; Wu, Y.; Li, Z.; Guo, X.; Zhang, M.; Zhang, S.; Hou, J. Macromolecules 2012, 45, 6923.
[9] Saadeh, H. A.; Lu, L.; He, F.; Bullock, J. E.; Wang, W.; Carsten, B.; Yu, L. ACS Macro Lett. 2012, 1, 361.

[10] Chen, J., Cao, Y. Acc. Chem. Res. 2009, 42, 1709

[11] Qin, R.; Li, W.; Li, C.; Du, C.; Veit, C.; Schleiermacher, H.; Andersson, M.; Bo, Z.; Liu, Z.; Inganäs, O.; Wuerfel, U.; Zhang, F. J. Am. Chem. Soc. 2009, 131, 14612.

[12] Hong, Y.; Wong, H.; Moh, L.; Tan, H. Chem. Commun. 2011, 47, 4920.

[13] Wang, B.; Tsang, S.; Zhang, W.; Tao, Y. Chem. Commun. 2011, $47,9471$.

[14] Jiang, Z.; Zhang, W.; Yao, H.; Yang, C., Cao, Y.; Qin, J.; Yu, G.; Liu, Y. J. Polym. Sci. Part A: Polym. Chem. 2009, 47, 3651.

[15] Wang, A. Chin. J. Mater. Res. 2012, 26(3), 327 (in Chinese). (王爱芬, 材料研究学报, 2012, 26(3), 327.)

[16] Cai, W.; Gong, X.; Cao, Y. Solar Energy Mater. Solar Cells 2010, 94, 114.

[17] Wang, E.; Ma, Z.; Zhang, Z.; Henriksson, P.; Inganäs, O.; Zhang, F.; Andersson, M. R. Chem. Commun. 2011, 47, 4908.

[18] Du, C.; Li, C.; Li, W.; Chen, X.; Bo, Z.; Veit, C.; Ma, Z.; Wuerfel, U.; Zhu, H.; Hu, W.; Zhang, F. Macromolecules 2011, 44, 7617.

[19] Zhang, Y.; Murphy, C. B.; Jones, W. E.; Jr. Macromolecules 2002, 35,630 .

[20] Qu, S.; Qin, C.; Islam, A.; Wu, Y.; Zhu, W.; Hua, J.; Tian, H.; Han, L. Chem. Commun. 2012, 48, 6972.

[21] Huo, L.; Zhang, S.; Guo, X.; Xu, F.; Li, Y.; Hou, J. Angew. Chem., Int. Ed. 2011, 50, 9697. 